\title{
Is Platelet-Activating Factor (PAF) a missing link for elucidating the mechanism of action of the coronavirus SARS-CoV-2 and explaining the side effects-complications of Covid-19 disease?
}

\author{
C.A.Demopoulos, Emeritus professor of Biochemistry \& Food Chemistry, \\ Faculty of Chemistry, National \& Kapodistrian University of Athens, \\ demopoulos@chem.uoa.gr
}

\begin{abstract}
Purpose of this article is to project from the point of view of the scientific data concerning PAF and as a new approach by involving PAF, in order to contribute to clarification and proposal a possible mechanism of action of the coronavirus SARSCoV-2 and to give a possible explanation for its observed side effects-complications of Covid-19 disease. The article is not intended to suggest any specific drugs, but clarifying the mechanism is the first step in illuminating the direction of prescribing the targets of medication that may be appropriate for the prevention, treatment, and cure of Covid-19 disease.

More specifically, the purpose of this article is to provide unequivocal evidence indicating that:

1 It would not be unexpected for PAFR to participate in the entry of coronavirus SARS-CoV-2 into the cell.

2 There is interdependence and relationship of the receptors ACER and PAFR, which means the involvement of PAF in the processes related to coronavirus SARS-CoV-2 as well as to its effects exercised by the coronavirus SARSCoV-2.

3 The receptors ACER, PAFR and TLRs are also involved in inflammation as well as in virus binding to stabilize and enter into the cell, and so they are "communicating vessels" with PAF being " a missing link"

4 Consequently there is a correlations of COVID-19 disease manifestations and biological characteristics with those caused by PAF, which shows the involvement of PAF in COVID-19 disease.
\end{abstract}

\section{Keywords:}

Platelet Activating Factor, PAF, SARS-CoV-2, Covid-19

\section{Introduction}

To date, a substantial body of evidence concerning the mechanism of action of the coronavirus SARS-CoV-2 and the Covid-19 disease has been published but the possible participation of PAF is largely ignored. There are, of course, few references to PAF in scientific journals and other media, but there is not a comprehensive- global- holistic proposal involving PAF in the mechanism of action of the coronavirus SARS-CoV-2 and in the side effects-complications of Covid-19 disease 
Specifically, until today, there are two papers concerning coronavirus SARSCoV-2 where PAF is reported [1,2]. However, there are also papers [3-6] that do not mention PAF, but they do mention and involve well-known PAF inhibitors [7]. Probable PAF implications with SARS-CoV-2 are also mentioned on youtube [8] and in an advertising prospect [9].

PAF is the most potent known lipid inflammatory factor, found in 1972 [10] In 1979 Demopoulos et al. elucidated its structure as a glyceryl-ether lipid (1-O-alkyl2-acetyl-sn-glycero-3-phosphocholine) described also its synthetic preparation [11].

After the elucidation of the structure of PAF, several international congresses, entirely focused on worldwide PAF research were organized every three years from 1983 until 2004. Since PAF research became interdisciplinary and expanded to virtually all areas of biochemistry and medicine, after 21 years the PAF congresses stopped being organized as much of the research surrounding PAF were disseminated at various international conferences. Nevertheless, attempts have been made to reignite these congresses and five years ago, on February 2015 in Tokyo Japan, the lipid mediator PAF communications were presented in special sessions at the "6th International Conference on Phospholipase A2 and Lipid Mediators" [12].

PAF is produced by many cells, eukaryotic, prokatiotic and plant cells as well as by tissues-organs in animals and humans. In addition it has many effects on cells and tissues-organs, which are usually the same cells and tissues-organs that produce it [13].

When the conditions on earth were initially intense, the cell-membrane phospholipids were mainly glyceryl-ether lipids, because the ether bond is a very stable bond and for the same reason mowedays halobacteria contain glyceryl monoand di-ether lipids. When the conditions became milder and until today in the course of evolution the lipids became glyceryl-ester lipids, where the ester bonds are much less stable bond than the ether bonds. However, some glyceryl-ether lipids of great biological importance remained and have been preserved to this day. One such glyceryl-ether lipid is PAF [14]. For this reason PAF exists and acts in all cell types, eukaryotic, prokaryotic and plants. Today, PAF is considered as "a universal cellular biological regulator", belonging to a family of molecules where PAF is the most active known member today.

PAF is implicated in almost all inflammatory conditions and plays a central role in inflammation. The figure 1 shows the main cellular and tissue-organs biological responses orchestrated by PAF. PAF participates in a vicious cycle where PAF triggers actions on cell/tissues-organs (e.g. cytokine secretion), which in turn retriggers PAF release [13]. 


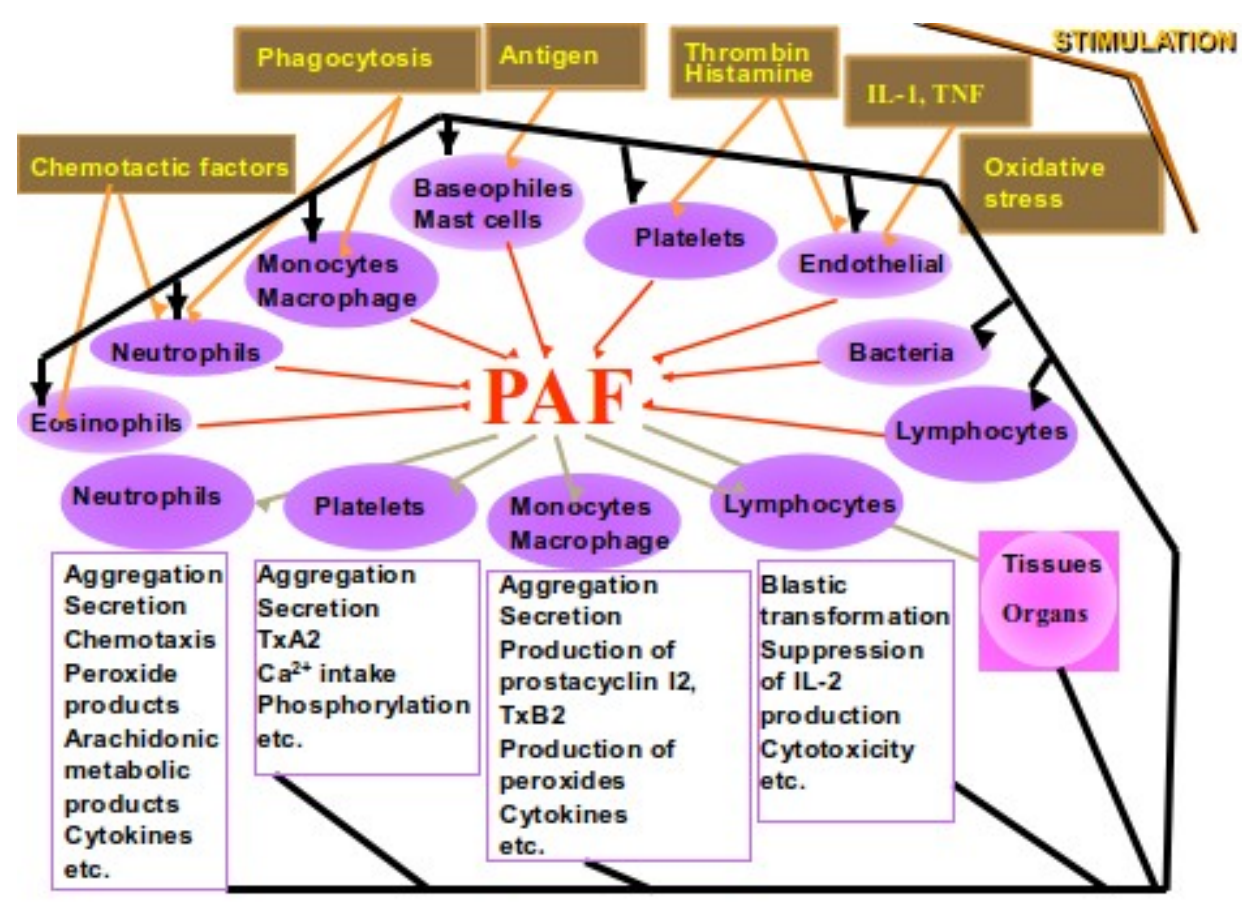

Figure1. The main cellular and tissue-organs biological responses orchestrated by PAF

It is pointed out that PAF is a "molecule signal- message " and therefore its actions (beneficial or harmful) are depending on the place, quantity and duration can be beneficial or harmful.

\section{The PAF receptor (PAFR) involvement in the entry of bacteria and viruses into the cells.}

As shown in the schematic representation of the main PAFR-induced signal transduction pathways (figure 2), the binding of PAF to its receptor initiates a 
cascade of intracellular events, which determines cell's response to PAF.

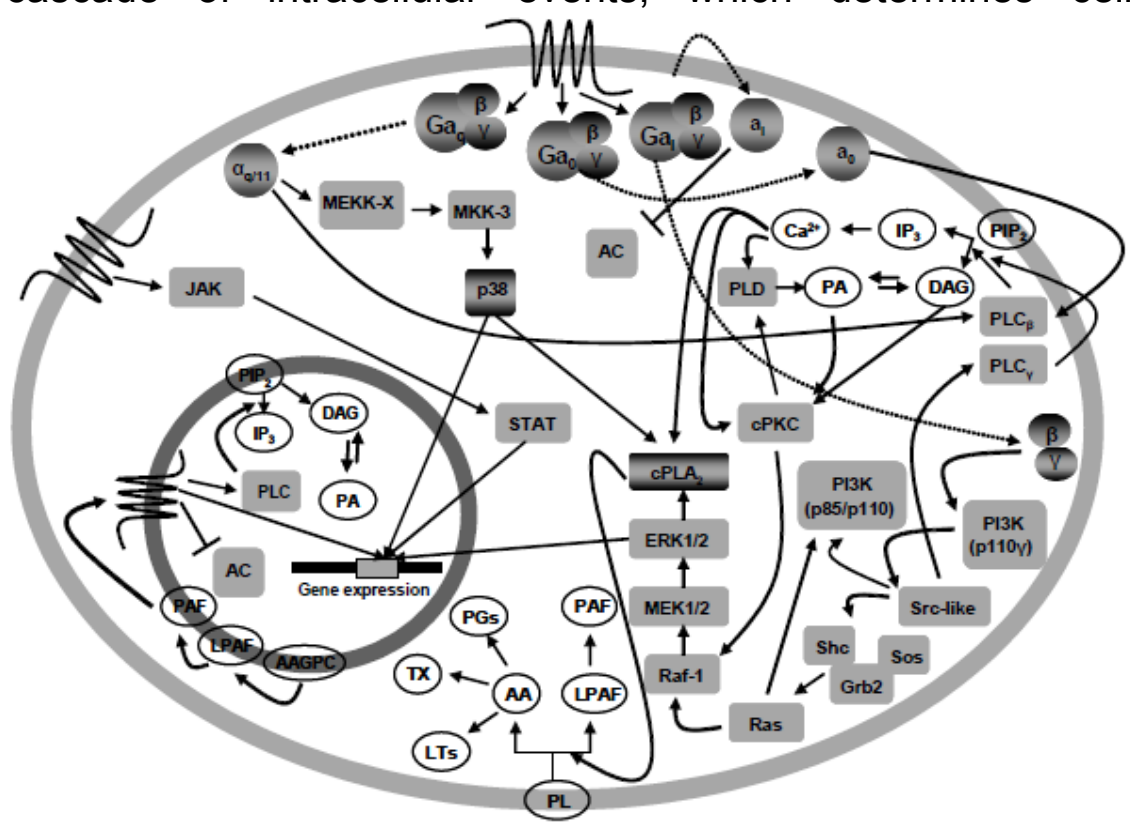

Figure 2: The main PAFR-induced signal transduction pathways.

Most of the well-characterized signal transduction pathways (PLC-mediated phosphoinositide turnover, $\mathrm{Ca}^{2+}$ mobilization, PI3K/Akt, MAPK, janus kinase (JAK)/signal transducer and activator of transcription protein STAT) are activated by PAF in a cell specific manner. PAFR is coupled with several isoforms of G-proteins. The type of cell and ligand determines the G-protein isoform(s) activated by PAFR each time. The ability of PAF to stimulate distinct signaling pathways via multiple Gproteins may explain the diverse biological responses of human cells to it [13].

PAF receptor is implicated in the entry not only of bacteria but also of the virus into the cell. It has been shown the entry of PAF in $\mathrm{H} 1 \mathrm{~N} 1$ and $\mathrm{H} 3 \mathrm{~N} 2$ viruses as well as the beneficial effects of PAF receptor inhibitors, such as the BN 52021 ginkgolide [15-18].

PAF and its receptor are known to be involved in viral infections such as HIV [19] with beneficial effects of PAF receptor inhibitors, such as the BN 52021 ginkgolide [20]. In addition, evidence in humans shows that highly active antiretroviral therapy with anti-PAF effects may attenuate PAF metabolism in the blood of HIV patients [21,22]. PAF-R antagonists have also been investigated for their anti-inflammatory effects in HIV-1-associated neurocognitive disorders [23].

PAF and its receptor are also known to be involved in dengue virus where PAFR blockade prevents more severe disease manifestations after infection with no increase in systemic viral titers [24], in respiratory syncytial virus [25] and in lung injury caused by influenza A [26], where PAF-R antagonism in mice suffering influenza $A$ induced protection against lung injury and mortality.

Consequently, it would not be unexpected for PAFR to participate in the entry of coronavirus SARS-CoV-2 into the cell.

After all, it is very likely that the coronavirus SARS-CoV-2 can enter into the cells in other ways and not only the Angiotensin-Converting Enzyme receptor is participated in the entry. 


\section{Angiotensin-Converting Enzyme receptor (ACER) and PAF}

It is known that ACE receptor participates in the regulation of blood pressure, but also in the regulation of the immune system and in the management of inflammation. But when these receptors malfunction, instead of acting protectively, they are involved in virus binding to stabilize and favor the entry of the coronavirus SARS-CoV-2 into the cells.

In pathological conditions associated with damage-dysfunction in ACE receptors, the literature shows the implication and primary role of PAF. The increased mortality from SARS-CoV-2 must also be due to these [27]. Disruption of receptor function leads also to immune system dysfunction, loss of the body's ability to keep the virus out of its cells, and susceptibility to infection, which appears to be the main aggravating factor in the development of the disease [28]. It has also been shown that the ACE receptor causes inflammation and is involved in inflammatory diseases [29].

In addition, Angiotensin produces PAF and PAF increases angiotensinconverting enzyme activity. Angiotensin increases intracellular calcium in glomerular mesangial cells and activates phospholipase A2, factors required for the formation of the lipid mediator of inflammation, PAF [30].

ACE is stimulated by PAF, and its activity in endothelial cells may be mediated by the PI-turnover pathway via changes in PLC activity and $\mathrm{IP}_{3}$-mediated $\mathrm{Ca}^{2+}$ release from intracellular stores [31].

When PAF is added to pulmonary artery endothelial cells, the conversion of angiotensin I to angiotensin II was enhanced about twofold at $10^{-6} \mathrm{~mol} / \mathrm{I} \mathrm{PAF} \mathrm{[32].}$

This explains why ACE is involved in the same diseases as PAF that is, ACE appear critical in a variety of disease states, including hypertension, diabetes, ageing, renal impairment, and cardiovascular disease [33]. Angiotensin-converting enzyme is a key enzyme in the renin-angiotensin-aldosterone system, converting angiotensin I to the vasoactive peptide angiotensin II. Besides being an important factor for normal regulation of blood pressure, ACE appears to be involved in the pathogenesis of atherosclerosis [34]. Also, ACE inhibitors have anti-inflammatory action and beneficial effects on the diseases where PAF is implicated [35]. Angiotensin-converting enzyme inhibitors reduce cardiovascular mortality in insulinresistant patients [36].

Angiotensin-converting enzyme inhibitors have anti-inflammatory activity [37]. The effects of statins, acetylsalicyclic acid and angiotensin-converting enzyme inhibitors on stroke prevention may partly be attributable to their profound antiinflammatory actions [38].

Captopril, and platelet-activating factor (PAF) antagonist prevent cardiac allograft vasculopathy in rats. Endogenous PAF and PAF-like compounds play a significant role [39].

Ramiprilat, prevents PAF-induced myocellular and endothelial injury in a neutrophil-perfused heart preparation [40].

Anti-ACE MoAb reveals PAF-induced changes in the status of the pulmonary ACE and therefore can be used for the studies of pathology of the pulmonary endothelium [41]. 
Statins and angiotensin receptor blockers have been effective in treating patients with sepsis, pneumonia and influenza, and a statin/ARB combination appeared to dramatically reduce mortality during the recent Ebola outbreak [42].

In fact, it seems that PAF and ACE have interdependent action. The interdependent effects of ACE and PAF-AH polymorphisms on the progression of IgA nephropathy might be more important than the effect of the individual polymorphisms [43].

Consequently, there is interdependence and relationship of the receptors ACER and PAFR, which means the involvement of PAF in the processes related to coronavirus SARS-CoV-2 as well as to its effects exercised by the coronavirus SARS-CoV-2.

\section{Toll Like Receptors (TLRs) and PAF}

TLRs is a transmembrane protein that belongs to the pattern recognition receptor family (PRR family), it recognizes molecules like lipopolysaccharide (LPS) and activate transcription factors like inducing proinflammatory cytokines expressions and Interferon

ACER, PAFR and TLRs, it is proven in the literature that they have common molecules that activate them, as molecules-agonists, one of which is PAF and the activation of all the above receptors can cause PAF release, too [44,30].

The aforementioned receptors ACER, PAFR and TLRs are also involved in inflammation as well as in virus binding to stabilize and enter into the cell, and so they are "communicating vessels" with PAF being a " the missing link".

Indicatively:

TLR4 is associated with infections but also with tissue damage, and this damagedependent pathway may be amplified in the acute stages of the infection. For this reason,TLR4-null mice are highly resistant to infection by the mouse adapted influenza A virus. In addition, TLR4 antagonists or specific anti-receptor antibodies show protection against influenza infections in mouse models. Another option in targeting host inflammatory response could then be targeting the TLR4 [45].

TLR4 engagement participate in platelet activation and amplify the secretion of proinflammatory mediators, providing an integrated view of platelet responses to pathogen- and host-derived agonists. This is potentially involved in platelet-mediated inflammation and cytokine-induced pathogenesis in dengue [46] where PAFR blockade prevents more severe disease manifestation after infection [47].

HIV-1 can be detected via interaction with TLRs (TLR7 and TLR8) by neutrophils which TLRs recognize subsequently viral nucleic acids and induce the generation of reactive oxygen species by MPO-derived oxidants that trigger NET formation and elimination of HIV-1. By this way, TLRs are on the one hand critical for host defense against pathogens and on the other hand contribute to the pathogenesis of autoimmunity [48].

Is highlighted that TLR agonists and antagonists are regarded as promising therapeutic agents for the treatment of sepsis, asthma, vaccine adjuvants, and autoimmunity 


\section{The side effects-complications of Covid-19 disease are related to actions of PAF and drugs that are inhibitors of PAF.}

Coronavirus SARS-CoV-2 infection induces impairment of multiple organ function. Even though the pathogenesis and etiology of COVID-19 disease remain unclear, this multiple organ function includes acute respiratory distress syndrome (ARDS), acute cardiac injury, acute kidney injury, liver impairment, neurological injury, gastrointestinal symptoms, immune system derangement e.t.c. But most cases of the disease caused by most pathogenic coronaviruses are mild with coagulation impairment [49].

Next, we describe the correlations of COVID-19 disease manifestations and biological characteristics with those caused by PAF.

Reactive oxygen species (ROS), which play a crucial role in the inflammatory response, produce PAF and PAF also produces ROS. For this reasons antioxidant properties may also be considered to reduce the cytokine storm induced by the vira infection, and antioxidative therapies are being considered for ameliorating cardiac injuries in critically ill COVID-19 patients [50].

Concerning SARS-CoV-1 has the ability of inducing production of compounds like oxidized-phospholipid (OxPL) both in humans and animals which induce cytokine production and acute lung injury via TLR4 [51]. It is reminded that, as we have published [52] that the oxidized lipids contain PAF and structural analogues of PAF that also show biological action PAF. For this reason SARS-CoV2 may induce acute lung injury and cytokine production, like that of IL-6 [45].

Neutrophil extracellular traps (NETs) is a neutrophil function that can be regarded as a double-edged sword: Neutralizing invading micro-organisms, but also can be harmful to the host, as its exposed by-products that are toxic to endothelial cells and parenchymal tissue [48]. Neutrophil activating factors may be derived from the host [e.g., platelet activating factor (PAF), leukotriene B4, interleukin-8 (IL-8)], or from pathogens [e.g., formylated peptide (fMLP) and lipopolysaccharide (LPS)]. NET-inducing factors identified to date include IL-8, PMA, bacteria, mycobacteria, fungi, protozoa, PAF, LPS, and M1 protein. COVID-19 disease manifestations and biological features correlate with those consecutive to uncontrolled NET formation causing various sterile or infectious diseases and NETs by-products may play a key role in COVID-19 pathogenesis. ROS act as key signaling molecule for NET formation. On the other hand, platelets promote inflammation and injury through critical interactions with neutrophils. It is well known that this tale place in sepsis where the activation of platelet TLR4 is a potent stimulus for the release of NETs by neutrophils. In addition, platelet accumulation on NETs may perpetuate platelet aggregation and activation and to promote coagulation in the lung microcirculation, leading to ischemic consequences. Cytokines released by neutrophils can also alter the membrane profile of vascular cells, and thus to participate in coagulation activation and to septic shock-induced endothelial dysfunction. In all the above cases and actions of NET, according to the literature, PAF is also involved.

A lot of biological actions of PAF after systematic or topical PAF administration, which are described in the literature, coincide to COVID-19 disease manifestations which are described by Jiancheng Zhang et al. [49]. For example, the following are common to PAF and COVID-19. Lungs: Pneumonia, acute respiratory distress syndrome. Cardiovascular system involvement: Arrhythmia, acute cardiac injury, shock. Liver impairment: Microvesicular steatosis, hepatocyte degeneration and necrosis, decrease in albumin level. Immune system derangement: 
Lymphocytopenia. Gastrointestinal symptoms: Diarrhea, oesophageal bleeding due to erosions and ulcers. Kidney injury: Increase in creatinine and urea nitrogen levels, proteinuria. Neurological injury: CNS symptoms, PNS symptoms, skeletal muscular symptoms. Coagulation disorders: Increase in D-dimers' and fibrinogen degradation products' levels, prolongation of prothrombin time. In addition development of Sepsis [62] and the most recently reported Kavasaki-like disease [53], in which PAF is implicated in, have been linked with Covid-19 infection.

A new therapeutic trend that has been consolidating in the last decade worldwide, opening new, more effective and safer ways to manage certain diseases, is multifunctional - hybrid drugs. They are suitable in the treatment of multifactorial diseases (many factors are involved in their manifestation) and these drugs act on more than one target. They come from the chemical combination of two or more different pharmaceutical molecule or pharmaceutical groups. On the other hand there is the use of "cocktail" drugs or a combination of different active molecules in one drug form. There is also the pleiotropy of the drugs. In pharmacology, pleiotropy includes all of a drug's actions other than those for which the agent was specifically developed.

Pharmaceutical objectives of the Covid-19 disease, since it is a multifactorial disease, should include pharmaceutical treatment which intend to inhibit the entry of virus into the cell, to have antiviral activity, and to inhibit the side effect-complications of Covid-19 disease. With other words the appropriate drug must be multifunctional hybrid drugs, or "Cocktail" of drugs or a combination of different active molecules in one drug form or drugs with the suitable pleiotropical actions for Covid-19 disease.

Indeed, there are many examples of the aforementioned drugs mentioned in the case of Covid-19 disease presenting anti-PAF action. Specific examples are listed below:

Statins, as mentioned, may be partly due to their beneficial effects on their deep anti-inflammatory effects [38, 42]. As we have published statins are in vitro and in vivo PAF inhibitors too [54].

The effects of PAF as well as of Covid-19 on organs and specifically to heart are well known. We have studied and demonstrated the inhibition of the actions of PAF (then called AGEPC) in the heart by the well-known Digoxin, presented as PAFinhibitor too [55].

PAF receptor is implicated in the entry not only of bacteria but of the virus into the cell. It has been shown the entry of PAF in $\mathrm{H} 1 \mathrm{~N} 1$ and $\mathrm{H} 3 \mathrm{~N} 2$ viruses as well as the beneficial effects of PAF receptor inhibitors, such as the BN 52021 ginkgolide. The beneficial effects of PAF receptor inhibitors, such as the BN 52021 ginkgolide in the entry of the virus into the cell has been reported [18]. It is also well know that BN 52021 ginkgolide is the best specific PAF receptor inhibitor. This beneficial effects of BN 52021 ginkgolide has been reported by our group in the case of another virus, the HIV [20].

Kaletra (Ritonavit, Lopinavir-r) have been studied by our group and we have published that Ritonavit, Lopinavir-r and Tenofovir-DF are the best inhibitors of both action and metabolism of PAF $[56,19]$. On the other hand, PAF is produced by Tat protein in the case of HIV. It is not unreasonable to be produced in the case of the coronavirus SARS-CoV-2, too.

The dysfunction of the ACE receptors used by the coronavirus SARS-CoV-2 to enter the body is due to a number of broader metabolic disorders, including metabolic disorders of vitamin D [28]. It is also suggested that increasing vitamin D intakes may reduce the risk of infections and, also, COVID-19 [57]. On the other 
hand, vulnerable groups need to maintain their vitamin $D$ status to reduce the risk of respiratory infections, including COVID-19 [6]. Adequate levels of vitamin D promote the innate immune response, since stimulation of TLR2 by a lipopeptide from an infectious organism results in increased expression of VDR, and then in the induction of cathelicidin. In addition, systemic factors such as vitamin D influence the adaptive immune response [58]. Our team has published that vitamin $D$ is an in vitro and in vivo PAF-inhibitor, too [59]. So PAF may be a link among Vit. D, TLR and ACE receptor.

Sepsis is also implicated in Covid-19 disease. In our study of antibiotics for sepsis, since sepsis according to the literature is related to PAF, we found that Clarithromycin and Azithromycin are the best inhibitors of both the action and the metabolism of PAF, but with Azithromycin to be the best of the two drugs $[60,61,62]$.

In relation to thrombotic complications in covid-19, it is well known that PAF is the strongest thrombotic factor and our unpublished data shows that almost all of the antiplatelet drugs are also PAF-inhibitors. Therefore, antiplatelet agents may be considered to treat COVID-19 [1].

Since a substantial body of evidence suggests how heparin can be beneficial in selected high-risk COVID-19 patients, today the development of heparin-based therapeutics is suggested. Indeed Rannuci et al. [63] showed that the use of an increased dose of low-molecular-weight heparin appeared to reduce the downstream thrombotic effects of the marked inflammatory response to COVID-19 as it seems to decrease the contribution of microvascular thrombosis in severely hypoxemic COVID patients. On the other hand Sasaki published [64] that low-molecular-weight heparin is a PAF-inhibitor.

One of the treatments tested in the randomized controlled trial of "RECOVERY" was low-dose dexamethasone. Dexamethasone is an old, inexpensive and proven drug that is a form of cortisone that has been used for many decades in many diseases and has a strong anti-inflammatory effect. Dexamethasone, till now, is the first drug to be shown to improve survival in COVID19 disease. Some examples of the relationship between dexamethasone and PAF are the following:

For many decades [65] it is well known that dexamethasone inhibits PAF-induced inflammation as well as [66] gastrointestinal damage induced by PAF. In addition, it has been shown [67] that PAF-induced granulocyte accumulation in guinea pig lung is inhibited by dexamethasone. Ibbotson and Wallace [68] studied the inhibitory effects of dexamethasone in endotoxic shock and its relationship to PAF synthesis in the gastrointestinal tract and lung. The onset of tissue damage and increases in vascular permeability in the gastrointestinal tract correlate temporally with changes in PAF synthesis and have previously been shown to be inhibited by PAF antagonists. But also pretreatment with dexamethasone resulted in a significant attenuation of endotoxin-induced hemoconcentration, hypotension and damage in the duodenum and stomach. Dexamethasone also significantly reduced PAF synthesis by the lung. Watanabe, Yagi et al. [69] studies indicated that the stimulation of neutrophil adherence to vascular endothelial cells by histamine and thrombin was inhibited by PAF antagonists and dexamethasone.

Our publication in 1988 [70], showed, for the first time, the implication of PAF in allergic rhinitis. Rupatadine-PAF inhibitor (the only drug-PAF inhibitor on the market, as RUPAFIN after RUpatadine PAF INhibitor) comes off in 2003 for allergic rhinitis [71]. This drug has together anti-histaminic and anti-PAF action. 
The development of multifunctional - hybrid drugs with anti-PAF activity is also studied in other cases, as in the case of anticancer drugs. It has been found from in vitro and in vivo experiments that co-administration of PAF inhibitors with anticancer drugs significantly improves their pharmacological action, because PAF is produced in the tumor area. Of course, the classic anti-cancer drugs (e.g. cis-platin) also have some anti-PAF action. Attempts are currently being made to design and manufacture complex compounds that also have anti-PAF action and are anti-cancer multifunctional - hybrid drugs [72].

Consequently there is a correlation of COVID-19 disease manifestations and biological characteristics with those caused by PAF, which shows the involvement of PAF in COVID-19 disease.

\section{Conclusion}

In conclusion, the goals for Covid-19 treatment, in addition to inhibiting virus reproduction, should be focused on two points:

The first is the entry of the virus into the body, where PAF receptor may be implicated in the entry of the virus into the cell.

The second concerns the treatment of SARS-CoV-2 -induced infection which induces impairment of multiple organ function. Going back to Figure 1, it is understandable that Covid-19 problems should be addressed on one hand by inhibiting inflammation and cytokine-induced pathogenesis as well as by inhibiting the direct actions of PAF exerted on cells and organs, but on the other hand by inhibiting the vicious cycle orchestrated by PAF where PAF triggers actions on cell / tissues-organs (eg cytokine secretion), which in turn re-triggers PAF release.

So the goals for Covid-19 treatment can be achieved by the use of pleiotropic drugs where their pleiotropic action includes the inhibition of PAF or multifunctional - hybrid drugs, or "Cocktail" of drugs that are also inhibitors of PAF.

\section{All the aforementioned data show that PAF constitutes an interesting example of an inflammatory molecule with promising role against coronavirus SARS- CoV-2.}

\section{References}

1. COVID-19 The Inflammation Link and the Role of Nutrition in Potential Mitigation. Nutrients (2020), 12, 1466; doi:10.3390/nu12051466.

loannis Zabetakis, Ronan Lordan, Catherine Norton and Alexandros Tsoupras.

2. Covid-19 induced superimposed bacterial infection. Journal of Biomolecular Structure and Dynamics, (2020)

DOI:10.1080/07391102.2020.1772110.

Mohamed A. Hendaus \& Fatima A. Jomha. 
3. Mechanisms of selected functional foods against viral infections with a view on COVID-19 Mini review.

Functional Foods in Health and Disease (2020), 5,195-209.

Alexander Haslberger G., Ursula Jacob, Berit Hippe, Heidrun Karlic.

4. Evidence that Vitamin D Supplementation Could Reduce Risk of Influenza and COVID-19 Infections and Deaths.

Nutrients (2020), 12, 988; doi:10.3390/nu12040988.

William B. Grant, Henry Lahore, Sharon L. McDonnell, Carole A. Baggerly, Christine B. French, Jennifer L. Aliano and Harjit P. Bhattoa.

5. Natural Polyphenol Engineering Field Crops based Diet to Promote Innate Immunity to Combat Covid-19 Disease.

agriRxiv (2020).

Zakir Hossain and Nashir Uddin.

6. Optimization of Vitamin D Status for Enhanced Immuno-protection Against Covid19.

Ir Med J (2020), 113, 58.

D.M. McCartney, D.G. Byrne.

7. Food Ingredients and Lipid Mediators.

Current Nutrition \& Food Science ( 2007), 3, 255-276.

Tzortzis Nomikos, Elizabeth Fragopoulou, Smaragdi Antonopoulou.

8. https://www.youtube.com/watch?v=KzKvlYwqQkE https://www.youtube.com/watch?v=qoJ4VDaGSfY

9. It Is Not Only the COVID-19 Virus that Is Dangerous. It Is How Our Body Reacts to It.

Belgian Family Physician. By Dr. Eric Beeth. Global Research, March 24, 2020.

Theme: Science and Medicine. A doctor's urgent appeal to look at blocking excess PAF-acether and strengthening the capillary bed in adults to diminish morbidity of the SARS-CoV-2.

10. LEUKOCYTE-DEPENDENT HISTAMINE RELEASE FROM RABBIT PLATELETS : THE ROLE OF IGE, BASOPHILS, AND A PLATELET-ACTIVATING FACTOR.

J.Exp.Med. (1972), 136, 1356.

J.Benveniste, P. M. Henson, C.G.Cochrane

11. Platelet-activating factor. Evidence for 1-O-alkyl-2-acetyl-sn-glyceryl-3phosphorylcholine as the active component (a new class of lipid chemical mediators).

J. Biol. Chem. (1979), 254, 9355-9358.

Demopoulos,C.A, Pinckard, R.N, Hanahan, D.J.

12. Forty Years Since the Structural Elucidation of Platelet-Activating Factor (PAF): Historical, Current, and Future Research Perspectives.

Molecules. (2019), 24(23). pii: E4414. doi: 10.3390/molecules24234414. 
Lordan R, Tsoupras A, Zabetakis I, Demopoulos CA.

13. PAF, a potent lipid mediator.

In " Bioactive Phospholipids. Role in Inflammation and Atherosclerosis" Editor:

Alexandros D. Tselepis, 2008: 85-134 ISBN: 978-81-7895-323-6, Trivandrum-695

023, Kerala, India

Smaragdi Antonopoulou, Tzortzis Nomikos, Haralabos C.Karantonis Elizabeth

Fragopoulou and Constantinos A. Demopoulos,

14. Kulikov et al. (1997) Biochemistry (Moscow) 62:1103

15. Binding of the non-typeable Haemophilus influenzae lipooligosaccharide to the PAF receptor initiates host cell signalling.

Cell Microbiol. (2001), 3, 525-536.

Swords, W. E., M. R. Ketterer, et al.

16. Platelet-Activating Factor Receptor Plays a Role in Lung Injury and Death Caused by Influenza A in Mice.

PLoS Pathog (2020). 6(11): e1001171. doi:10.1371/journal.ppat.1001171.

Cristiana C. Garcia, Remo C. Russo, Rodrigo Guabiraba, Caio T. Fagundes, Rafael B. Polidoro,Luciana P. Tavares, Ana Paula C. Salgado, Geovanni D. Cassali, Lirla^ndia P. Sousa, Alexandre V.Machado, Mauro M. Teixeira.

17. Expression and DNA Microarray Analysis of a Platelet Activating Factor-Related Molecule in Severe Pneumonia in Mice Due to Influenza Virus and Bacterial CoInfection.

Jpn. J. Infect. Dis., (2009), 62, 6-10.

Masafumi Seki, Kosuke Kosai, Atsuko Hara, Yoshifumi Imamura, Shigeki Nakamura,

Shintaro Kurihara, Koichi Izumikawa, Hiroshi Kakeya, Yoshihiro Yamamoto, Katsunori Yanagihara, Yoshitsugu Miyazaki, Hiroshi Mukae, Takayoshi Tashiro, and Shigeru Kohno.

18. Anti-influenza virus activity of Ginkgo biloba leaf extracts.

J Nat Med (2013), 67, 636-642.

Haruyama, T. and K. Nagata.

19. The Role of Platelet-Activating Factor in Chronic Inflammation, Immune Activation, and Comorbidities Associated with HIV Infection.

AIDS Rev. (2015), 17, 191-201.

Theodoros Kelesidis, Vasiliki Papakonstantinou, Paraskevi Detopoulou, Elizabeth Fragopoulou, Maria Chini, Marios C. Lazanas and Smaragdi Antonopoulou.

20. In vivo effects of a Ginkgo biloba extract on Platelet Activating Factor metabolism in two asymptomatic HIV-infected patients .

European Journal of Inflammation (2011), 9,107-116

A.B. Tsoupras, M. Chini, N. Tsogas, N. Mangafas, C.A. Demopoulos and M.C. Lazanas.

21. In vivo effect of two first-line art regimens on inflammatory mediators in male HIV patients. 
Lipids Health Dis. (2014), 13, 90.

Papakonstantinou, V.D.; Chini, M., Mangafas, N., Stamatakis, G.M., Tsogas, N., Tsoupras, A.B., Psarra, K., Fragopoulou, E., Antonopoulou, S., Gargalianos, P., et al.

22. Anti-platelet-activating factor effects of highly active antiretroviral therapy (HAART): A new insight in the drug therapy of HIV infection?

AIDS Res. Hum. Retrovir. (2008), 24, 1079-1086.

Tsoupras, A.B., Chini, M., Tsogas, N., Fragopoulou, E., Nomikos, T., Lioni, A., Mangafas, N., Demopoulos, C.A., Antonopoulou, S., Lazanas, M.C.

23. Development of a platelet-activating factor antagonist for HIV-1associated neurocognitive disorders. J. Neuroimmunol. (2009), 213, 47-59.

Eggert, D., Dash, P.K., Serradji, N., Dong, C.-Z., Clayette, P., Heymans, F., Dou, H., Gorantla, S., Gelbard, H.A., Poluektova, L., et al.

24. Essential role of platelet-activating factor receptor in the pathogenesis of Dengue virus infection.

PNAS (2009), 106, 14138-14143.

Danielle G. Souzaa, Caio T. Fagundesa, Lirlandia P. Sousaa, Flavio A. Amarala, Rafael S. Souzaa, Adriano L. Souzaa,Erna G. Kroonb, Daniela Sachsa, Fernando Q. Cunhac, Eugenij Bukind, Alena Atrasheuskayad, George Ignatyeve and Mauro M.

Teixeira.

25. Respiratory syncytial virus infection of human mononuclear phagocytes stimulates synthesis of platelet-activating factor.

J. Biol. Chem. (1991), 266, 5472-5479.

Villani, A., Cirino, N., Baldi, E., Kester, M., McFadden, E., Panuska, J.

26. Platelet-activating factor receptor plays a role in lung injury and death caused by influenza a in mice.

PLoS Pathog. (2010), 6, e1001171.

Garcia, C.C., Russo, R.C., Guabiraba, R., Fagundes, C.T., Polidoro, R.B., Tavares, L.P., Salgado, A.P.C., Cassali, G.D., Sousa, L.P., Machado, A.V., et al.

27. Report sulle caratteristiche dei pazienti deceduti positivi a COVID-19 in Italia II presente report è basato sui dati aggiornati al 20 Marzo 2020.

https://www.epicentro.iss.it/coronavirus/bollettino/Report-COVID-2019_20_marzo.pdf

28. Comparative genetic analysis of the novel coronavirus (2019-nCoV/SARS-CoV2) receptor ACE2 in different populations.

Cell Discovery (2020) volume 6, Article number: 11

https://www.nature.com/articles/s41421-020-0147-1

Yanan Cao, Lin Li, Zhimin Feng, Shengqing Wan, Peide Huang, Xiaohui Sun, Fang

Wen, Xuanlin Huang, Guang Ning \& Weiqing Wang. Naturer.

29. Inflammation and Atherosclerosis: The Role of Renin-Angiotensin System and Its Inhibition.

Kardiologiya (2010), 50, 10.

Partigulova, A. S. and V. G. Naumov, 
30. Angiotensin II Causes Formation of Platelet Activating Factor in Cultured Rat Mesangial Cells.

Circ Res. (1989), 64,1224-9.

Neuwirth R, Satriano JA, DeCandido S, Clay K, Schlondorff D.

31. Mechanism of increased angiotensin-converting enzyme activity stimulated by platelet-activating factor.

Biochim Biophys Acta. (1990), 1052, 503-8.

Kawaguchi $\mathrm{H}$, Sawa $\mathrm{H}$, Yasuda $\mathrm{H}$.

32. Platelet-activating factor stimulates angiotensin converting enzyme activity.

J Hypertens. (1990), 8, 173-7.

Kawaguchi $\mathrm{H}$, Sawa $\mathrm{H}$, lizuka K, Yasuda $\mathrm{H}$.

33. Angiotensin-Converting Enzyme 2 (ACE2) Is a Key Modulator of the Renin Angiotensin System in Health and Disease.

Int J Pept. 2012;2012:256294. doi: 10.1155/2012/256294. Epub 2012 Mar 20. Tikellis C, Thomas MC.

34. Angiotensin-Converting Enzyme Effects of Smoking and Other Risk Factors for Cardiovascular .

Linköping: Linköping University Electronic Press , 2009. , p. 67.

Linköping Studies in Health Sciences. Thesis, ISSN 1100-6013 ; 92

35. Impact of Combination Medical Therapy on Mortality in Vascular Surgery Patients.

Journal of Hospital Medicine (2010), 5, 218-225.

Barrett, T. W., K. Newton, et al.

36. Insulin Resistance and Atherosclerosis.

Endocrinology and Metabolism Clinics of North America (2008), 37, 603.

Razani, B., M. V. Chakravarthy, et al.

37. Inflammation in atherosclerosis and psoriasis: common pathogenic mechanisms and the potential for an integrated treatment approach.

British Journal of Dermatology (2008), 159, 10-17.

Spah, F.

38. Inflammation and atherosclerosis - Novel insights into plaque formation and destabilization.

STROKE (2006) 37, 1923-1932

Stoll, G. and M. Bendszus.

39. Captopril, and platelet-activating factor (PAF) antagonist prevent cardiac allograft vasculopathy in rats: role of endogenous PAF and PAF-like compounds.

J Heart Lung Transplant (1999) 18, 470-477.

Crawford SE, Huang L, Hsueh W, Takami H, Gonzalez-Crussi F, Backer CL, Mu Y, Liu $\mathrm{H}$, Mavroudis $\mathrm{C}$. 
40. Ramiprilat, prevents PAF-induced myocellular and endothelial injury in a neutrophil-perfused heart preparation.

Agents Actions Suppl (1992) 38 ( Pt 3): 209-216.

Schrör K, Felsch A.

41. Systemic administration of platelet-activating factor in rat reduces specific pulmonary uptake of circulating monoclonal antibody to angiotensin-converting enzyme.

Lung. (1992), 170, 349-58.

Atochina EN, Hiemisch HH, Muzykantov VR, Danilov SM.

42. Treating the host response to emerging virus diseases: lessons learned from sepsis, pneumonia, influenza and Ebola.

Ann Transl Med. (2016), 4, 421. doi: 10.21037/atm.2016.11.03.

David S. Fedson.

43. Interdependent effect of angiotensin-converting enzyme and platelet-activating factor acetylhydrolase gene polymorphisms on the progression of immunoglobulin $A$ nephropathy.

Clinical Genetics (2002), 2, 128-134.

Yoon, H. J., H. Kim, et al.

44. Mediterranean diet and its protective mechanisms against cardiovascular disease: An insight into Platelet Activating Factor (PAF) and diet interplay.

Annals of Nutritional Disorders \& Therapy (2015), 2, 1-10 ISSN:2381-8891

Detopoulou P, Demopoulos C.A, Karantonis H.C, and Antonopoulou S.

45. SARS-CoV-2 and COVID-19: is interleukin-6 (IL-6) the 'culprit lesion' of ARDS onset? What is there besides Tocilizumab? SGP130Fc

Cytokine: X (2020), doi: https://doi.org/10.1016/ j.cytox.2020.100029

Giuseppe Magro.

46. Inflammatory signaling in dengue-infected platelets requires translation and secretion of nonstructural protein 1.

Blood Adv. (2020), 4, 2018-2031. doi: 10.1182/bloodadvances.2019001169. Anna Cecíllia Quirino-Teixeira, Stephane Vicente Rozini, Giselle BarbosaLima, Diego Rodrigues Coelho, Pedro Henrique Carneiro, Ronaldo MohanaBorges, Patrícia T Bozza, Eugenio D Hottz.

47. Essential role of platelet-activating factor receptor in the pathogenesis of Dengue virus infection.

Proc Natl Acad Sci U S A. (2009), 106, 14138-14143.

Published online 2009 Jul 30. doi: 10.1073/pnas.0906467106.

Danielle G. Souza, Caio T. Fagundes, Lirlandia P. Sousa, Flavio A. Amaral, Rafael

S. Souza, Adriano L. Souza, Erna G. Kroon, Daniela Sachs, Fernando Q. Cunha, Eugenij Bukin, Alena Atrasheuskaya, George Ignatyev, and Mauro M. Teixeira.

48. NETs by-products and extracellular DNA may play a key role in COVID-19 pathogenesis: incidence on patient monitoring and therapy. Preprints (2020), 2020040238 (doi: 10.20944/preprints202004.0238.v1). 
Alain R. Thierry and Benoit Roch.

49. Current status of potential therapeutic candidates for the COVID-19 crisis. Behavior, and Immunity (2020), https://doi.org/10.1016/j.bbi.2020.04.046. Jiancheng Zhang, Bing Xie, Kenji Hashimoto Brain.

50. An anti-oxidative therapy for ameliorating cardiac injuries of critically ill COVID19-infected patients.

Int. J. Cardiol. (2020). Available online: https://www.ncbi.nlm.nih.gov/pubmed/ 32321655 (accessed on 1 April 2020).

Wang, J.-Z.; Zhang, R.-Y.; Bai, J.

51. Identification of oxidative stress and Toll-like receptor 4 signaling as a key pathway of acute lung injury.

Cell 133 (2008) 235-249.

Y. Imai, K. Kuba, G.G. Neely, et al.,

52. Platelet-Activating Factor formation during oxidative modification of low-dencity lipoprotein when PAF-acetylhydrolase has been inactivated.

Biochim. Biophys. Acta (1994) 1212, 353

T.A.Liapikos, S.Antonopoulou, S.-A.Karabina, D.C.Tsoukatos, C.A.Demopoulos and A.D.Tselepis.

53. Elevated Levels of Platelet Activating Factor and Its Acetylhydrolase Indicate High Risk of Kawasaki Disease.

Journal of Interferon \& Cytokine Research (2020), 40,

https://doi.org/10.1089/jir.2019.0141

Lunyu Yi, Jing Zhang, Jiarong Zhong and Yuqiang Zheng.

54. In vitro and in vivo effects of statins on Platelet Activating Factor (PAF) and its metabolic enzymes.

Angiology (2011), 62, 209-218.

Nektaria Tsantila,Alexandros B Tsoupras,Elizabeth Fragopoulou,Smaragdi Antonopoulou, Christos latrou, Constantinos A Demopoulos .

55. Study of digoxin as inhibitor of the in vivo effects of acetyl glyceryl ether phosphorylcholine (AGEPC) in mice.

Life Sciences (1988), 42, 623.

D.Kelefiotis, E.Lanara, C.Vakirtzis-Lemonias, A.Siafaka, M.Mavris, M.Lazanas and C.A.Demopoulos.

56. Anti - Platelet Activating Factor effects of Highly Active Anti-Retroviral Therapy (HAART): A new insight in the drug therapy of HIV infection?

AIDS Researchand Human Retroviruses (2008), 24,1079-1086.

Alexandros B. Tsoupras, Maria Chini, Nickolaos Tsogas, Elizabeth Fragopoulou, Tzortzis Nomikos, Athina Lioni, Nikolaos Mangafas, Constantinos A. Demopoulos, Smaragdi Antonopoulou, Marios C. Lazanas.

57. Evidence that vitamin D supplementation could reduce risk of influenza and COVID-19 infections and deaths. 
Nutrients (2020), 12, 988.

Grant,W.B.; Lahore, H.; McDonnell, S.L.; Baggerly, C.A.; French, C.B.; Aliano, J.L.; Bhattoa, H.P.

58. Vitamin D Metabolism, Mechanism of Action, and Clinical Applications Chem Biol. (2014), 21, 319-329.

Daniel D. Bikle.

59. Paricalcitol effects on activities and metabolism of platelet activating factor and on inflammatory cytokines in hemodialysis patients.

Int. J. Artif. Organs (2013), 36, 87-96. DOI:10.5301/ijao.5000187.

Verouti SN, Tsoupras AB, Alevizopoulou F, Demopoulos CA, latrou C.

60. In vitro anti-inflammatory and anti-coagulant effects of Antibiotics towards Platelet Activating Factor and Thrombin.

Journal of Inflammation (2011), 8,17. doi:10.1186/1476-9255-8-17.

Alexandros B Tsoupras, Maria Chini, Nickolaos Tsogas, Athina Lioni, George Tsekes, Constantinos A Demopoulos and Marios C Lazanas.

61. Anti-Platelet Acticating Factor (PAF). Effects of Antibiotics: A New Insight in Therapy of Sepsis?

Eur. Congr. Of Clinical Microbiology and Infectious Diseases 19-22 April 2008, Barcelona, Spain. Clinical Microbiology and Infection 14, s7 - 2008 - (S1-S815) A.B.Tsoupras, M.Chini, N.Tsogas,A. Lioni,G. Tsekes , C.A.Demopoulos, M.C.Lazanas.

62. Involvement of platelet-activating factor (PAF) in septic shock and priming as indicated by the effect of hetrazepinoic PAF antagonists.

Lipids (1991), 26, 1369-1373.

Heuer H.O.

63. The procoagulant pattern of patients with COVID-19 acute respiratory distress syndrome.

J. Thromb. Haemost. (2020). doi: 10.1111/jth.14854.

Ranucci, M., Ballotta, A., Di Dedda, U., Bayshnikova, E., Dei Poli, M., Resta, M., Falco, M., Albano, M.,

Albano, G., Menicanti, L.

64. M.,Br.J.Pharmacol.(1993),110:107-112.

Sasaki

65.Dexamethasone inhibits platelet activating factor-induced inflammation in the paw but not the pleural cavity of rats.

Eur J Pharmacol (1988), 150, 131-135.

Peers, S. H.

66.Gastrointestinal damage induced by platelet-activating factor. Inhibition by the corticoid, dexamethasone.

Dig Dis Sci (1988), 33, 225-232.

Wallace, J. L. and B. J. Whittle 
67.Inhibition of PAF-, LPS-, and cytokine-induced granulocyte accumulation in guinea pig lung by dexamethasone: evidence that inhibition of IL-5 release is responsible for the selective inhibition of eosinophilia by glucocorticoids in guineapigs.

Inflamm Res (1996), 45, 166-170

Whelan, C. J.

68. Inhibitory effects of dexamethasone in endotoxic shock and its relation to PAFacether synthesis in the gastrointestinal tract and lung.

J Lipid Mediat (1989), 1, 273-282.

Ibbotson, G. C. and J. L. Wallace

69.Stimulation of neutrophil adherence to vascular endothelial cells by histamine and thrombin and its inhibition by PAF antagonists and dexamethasone.

Br J Pharmacol (1991), 102, 239-245.

Watanabe, M., M. Yagi, et al.

70. PAF of biological fluids in disease: Blood levels in allergic rhinitis.

Haemotologica (1988) 3, 379.

K.Labrakis-Lazanas, M.Lazanas, S.Koussissis, S.Tournis and C.A.Demopoulos.

71. RUPATADINE. A new selective histamine $\mathrm{H} 1$ receptor and platelet activation factor (PAF) antagonist.

Drugs of Today (2003) 39, 451.

I.Izquierdo, M.Merlos, J.Garcia-Rafanell.

72. A review on platelet activating factor inhibitors: Could a new class of potent metal-based anti-inflammatory drugs induce anticancer properties?

Bioinorg. Chem. Appl. (2017), 2017.

Papakonstantinou, V.D., Lagopati, N., Tsilibary, E.C., Demopoulos, C.A., Philippopoulos, A.I. 